\title{
An inverse method for color uniformity in white LED spotlights
}

\author{
Corien Prins ${ }^{1 *}$, Jan ten Thije Boonkkamp ${ }^{1}$, Teus Tukker ${ }^{2}$ and Wilbert IJzerman ${ }^{3}$
}

\author{
"Correspondence: c.r.prins@tue.nl \\ ${ }^{1}$ Centre for Analysis, Scientific \\ computing and Applications \\ (CASA), Technische Universiteit \\ Eindhoven, P.O. Box 513, $5600 \mathrm{MB}$ \\ Eindhoven, The Netherlands \\ Full list of author information is \\ available at the end of the article
}

\begin{abstract}
Color over Angle (COA) variation in the light output of white phosphor-converted LEDs is a common problem in LED lighting technology. In this article we propose an inverse method to design an optical element that eliminates the color variation for a point light source. The method in this article is an improved version of an earlier method by the same authors, and provides more design freedom than the original method. We derive a mathematical model for color mixing in a collimator and present a numerical algorithm to solve it. We verify the results using Monte-Carlo ray tracing.
\end{abstract}

\section{Introduction}

LED is a rising technology in the field of lighting. In the past, LEDs were only suitable as indicator lights, but the enormous improvements in energy efficiency, cost and light output now allow the use of LEDs for lighting applications [1]. Additionally, LED lighting benefits from low maintenance cost and long lifetime.

Because LED is a rising technology, companies and researchers are constantly searching for methods to reduce the production cost and increase the efficiency, light output and light quality of LED-based lamps. An important issue for white LED lamps is color variation of the emitted light. This is caused by color variation in the light output of the most common type of white LED, the phosphor-converted LED. This type of LED consists of a blue LED with on top a so-called phosphor layer which converts part of the blue light into yellow and red. The resulting output is white light. The distance that a light ray travels through the phosphor depends on the angle of emission. As a result, the light emitted normal to the LED surface is more bluish, while the light emitted nearly parallel to the surface is more yellowish [2, pp.353-357]. This phenomenon is called Color over Angle (CoA) variation.

A lot of research has been done to reduce this color variation. Introduction of bubbles in the phosphor layer causes scattering of light, reducing the color variation [3]. Another common method is the application of a dichroic coating on the LED [4]. However, these methods reduce the efficiency of the LED and increase the production costs. Wang et al. [5] proposed a modification of the optics on the LED to improve the color uniformity. In the case of a spot light, the LED is combined with a collimator. A collimator is an optical component that reduces the angular width of the light emitted by the LED. A common technique is to add a microstructure on top of the collimator. However, this microstructure introduces extra costs in the production process of the collimator, makes the collimator look unattractive and broadens the light beam.

๑2014 Prins et al.; licensee Springer. This is an Open Access article distributed under the terms of the Creative Commons Attribution License (http://creativecommons.org/licenses/by/2.0), which permits unrestricted use, distribution, and reproduction in any medium, provided the original work is properly cited. 
None of the methods mentioned above rigorously solve the problem of color variation, and all methods reduce the efficiency of the optical system. In earlier work [6], we introduced an inverse method to design a specific type of collimator, the so-called TIR (total internal reflection) collimator. The TIR collimator designed with this method mixes light from a point source such that the color variation is completely eliminated. The collimator requires no microstructures nor scattering techniques. However, the inverse method left very little design freedom for optical designers. An optical designer wants to influence the height and width of the collimator, for example, to fit it into the available space in a lamp design. Also, optical designers want a color mixing collimator which resembles a standard collimator as closely as possible. The inverse method introduced in this paper is an improvement of the method introduced in [6]. The collimator has three free surfaces instead of two. As a result, the improved method offers more design freedom, and it is nearly impossible to distinguish the resulting collimator with the naked eye from a collimator without color correction.

The contents of this paper is the following. First we give a thorough introduction to inverse methods for optical systems and the theory of color mixing in Section 2. In Section 3 we explain the improved inverse method. Section 4 describes three examples where the new method is used. Finally, we end with concluding remarks in Section 5.

\section{Design of a TIR collimator using inverse methods}

A TIR collimator is a rotationally symmetric lens, usually made of a transparent plastic like polycarbonate (PC) or polymethyl methacrylate (PMMA), that is used to collimate the light of an LED into a compact beam. A profile of a TIR collimator can be seen in Figure 1. The design procedure using inverse methods consists of two steps: first we choose a relation between the angles $t$ of rays leaving the LED and the angles $\theta$ of rays leaving the collimator, the so-called transfer functions. Subsequently we use these transfer functions to calculate the free surfaces of the TIR collimator such that the light is redirected according to the relation defined by the transfer functions. In Figure 1 these free surfaces are denoted by $\mathrm{A}, \mathrm{B}$ and $\mathrm{C}$.

\subsection{Source and target intensities}

The first requirement that determines the choice of the transfer functions is the intensity pattern of the light emitted from the TIR collimator. Let $I(t, u)[\mathrm{lm} / \mathrm{sr}]$ be the intensity

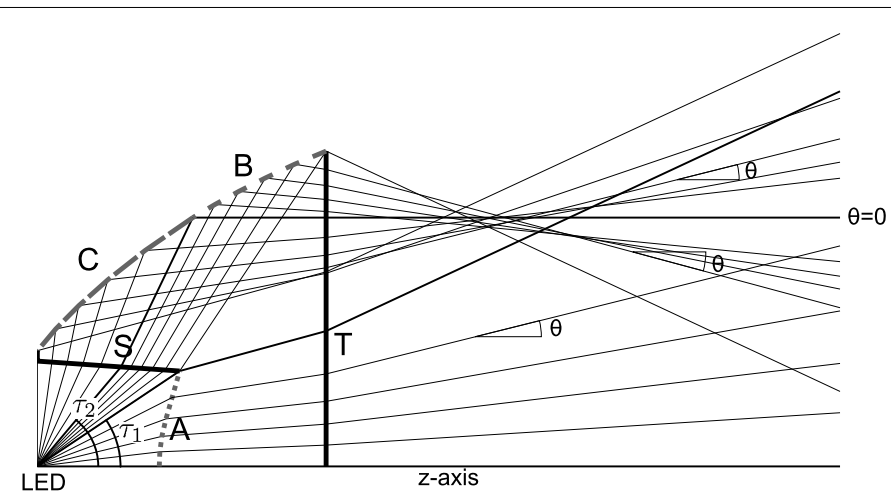

Figure 1 Profile of a TIR collimator. A full TIR collimator can be obtained by rotating the profile around the $z$-axis. Surface $B$ and $C$ are separated by the ray with angle $\theta=0$. 
distribution of the light source. The unit $\mathrm{lm}$ stands for lumen, and is the unit to denote energy flux corrected for the sensitivity of the eye at different wavelengths, and sr stands for steradian, the unit of solid angle. The angle $t \in[0, \pi / 2]$ is the angle with respect to the $z$-axis (inclination), and $u \in[0,2 \pi)$ is the angle that rotates around the $z$-axis (azimuth). Because of the symmetry of the system, the intensity $I(t, u)$ is independent of $u$ and denoted by $I(t)$. We introduce an effective intensity $\mathcal{I}(t)$, which is the flux per rad through the circular strip $[t, t+\mathrm{d} t]$ on the unit sphere divided by $2 \pi$. We calculate $\mathcal{I}(t)$ by integrating $I(t)$ over the angle $u$ :

$$
\mathcal{I}(t)=\frac{1}{2 \pi} \int_{0}^{2 \pi} I(t) \sin (t) \mathrm{d} u=I(t) \sin (t) .
$$

The effective intensity has unit $[\mathrm{lm} / \mathrm{rad}]$. For an LED, the effective intensity is typically positive for $t \in(0, \pi / 2)$.

The light emitted from the TIR collimator has a desired pattern in the far field, meaning that the TIR collimator itself can be considered a point source. The desired intensity profile is denoted by $G(\theta, \phi)[\mathrm{lm} / \mathrm{sr}]$, where $\theta \in\left[0, \theta_{\max }\right]$ is the inclination for some maximum inclination angle $0<\theta_{\max } \leq \pi / 2$, and $\phi \in[0,2 \pi)$ is the azimuth. We only consider intensity profiles that are rotationally symmetric and thus independent of $\phi$. Integration over the angle $\phi$ results in an effective intensity $\mathcal{G}(\theta)=\sin (\theta) G(\theta)[\mathrm{lm} / \mathrm{rad}]$. A more in-depth discussion of effective intensity distributions can be found in Maes [7]. The target intensity is multiplied by a constant $c>0$ such that we have conservation of luminous flux for the optical system:

$$
\int_{0}^{\pi / 2} \mathcal{I}(t) \mathrm{d} t=c \int_{0}^{\theta_{\max }} \mathcal{G}(\theta) \mathrm{d} \theta
$$

The angular space $[0, \pi / 2]$ of the light emitted by the LED is partitioned into $N \in \mathbb{N}$ segments $\left[\tau_{i-1}, \tau_{i}\right], i=1,2, \ldots, N$. For each segment we define a transfer function $\eta_{i}$ : $\left[0, \theta_{\max }\right] \rightarrow\left[\tau_{i-1}, \tau_{i}\right] \subset[0, \pi / 2]$. For a certain $\theta, \eta_{i}(\theta)$ gives the emission angle $t$ of the LED in $\left[\tau_{i-1}, \tau_{i}\right]$. We choose each transfer function to be strictly monotonic and thus invertible. The luminous flux emitted from the collimator in the interval $[\theta, \theta+\mathrm{d} \theta]$ must be equal to the sum over $i$ of the luminous fluxes emitted from the source in each interval $\left[\eta_{i}(\theta), \eta_{i}(\theta+\mathrm{d} \theta)\right]$. This leads to the following relation:

$$
\sum_{i=1}^{N} \sigma_{i} \mathcal{I}\left(\eta_{i}(\theta)\right) \eta_{i}^{\prime}(\theta)=c \mathcal{G}(\theta),
$$

where $\sigma_{i}=-1$ for monotonically decreasing transfer functions and $\sigma_{i}=1$ for monotonically increasing transfer functions.

\subsection{Color mixing}

The second requirement on transfer functions is related to the color of the resulting beam from the collimator. First we give a short introduction to the theory of color perception, then we derive an ordinary differential equation describing the color of the beam.

Color perception is described extensively in $[8,9]$. The human perception of a beam of light can be fully described by its luminous flux (in $\mathrm{lm}$ ) and the two so-called chromaticity coordinates $0<x, y<1$. There is a simple rule to calculate the chromaticity coordinates 


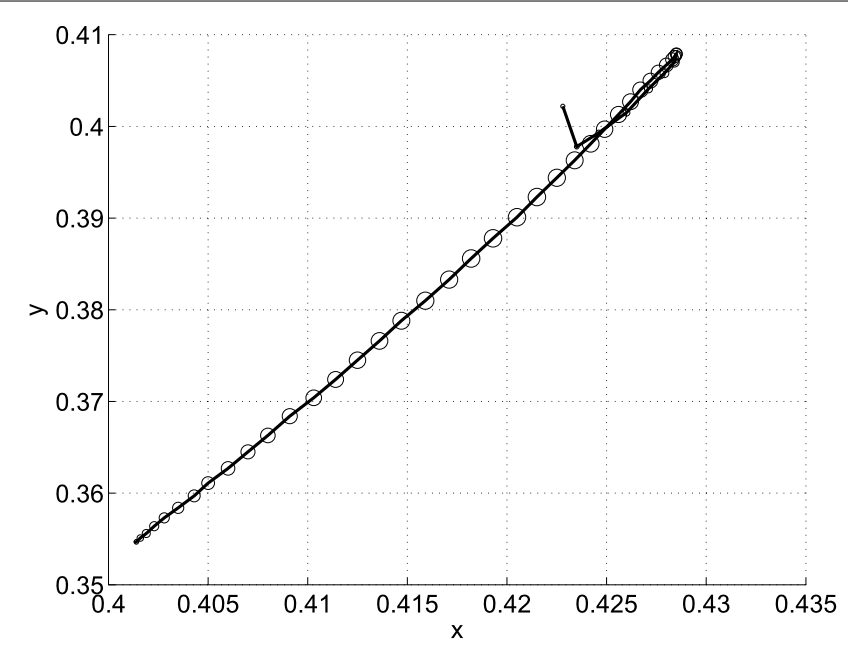

Figure 2 Scatter plot of the measured $x$ and $y$ chromaticity coordinates of the LED used in the numerical experiments in this article. The size of the circles corresponds to the effective intensity. The measured data in the lower left corner correspond small angles of $t$. The measured data for values of $t$ close to $\pi / 2$ around $(0.423,0.4)$ are unreliable because of the low light intensities, causing the irregularity.

$(x, y)$ of the beam resulting from mixing two beams of light with luminous fluxes $L_{1}$ and $L_{2}$ and chromaticity coordinates $\left(x_{1}, y_{1}\right)$ and $\left(x_{2}, y_{2}\right)$, respectively:

$$
\begin{aligned}
& x=\frac{x_{1} L_{1} / y_{1}+x_{2} L_{2} / y_{2}}{L_{1} / y_{1}+L_{2} / y_{2}}, \\
& y=\frac{L_{1}+L_{2}}{L_{1} / y_{1}+L_{2} / y_{2}} .
\end{aligned}
$$

The resulting chromaticity coordinates are weighted averages of the chromaticity coordinates of the original beam with weights $L_{1} / y_{1}$ and $L_{2} / y_{2}$. Note that a point $(x, y)$ is on the straight line segment between $\left(x_{1}, y_{1}\right)$ and $\left(x_{2}, y_{2}\right)$.

The chromaticity coordinates of the light emitted from an LED are not constant, but depend on the angle of emission $t$ and are described by functions $x(t)$ and $y(t)$. From measured data we have observed an approximate linear relationship between $x(t)$ and $y(t)$, see Figure 2. From the color mixing rule, we conclude that if we mix light from different angles of the LED into a single beam with color coordinates $\left(x_{T}, y_{T}\right)$, these coordinates must be on the straight line segment relating $x(t)$ and $y(t)$. Therefore, given $y_{T}$, the chromaticity coordinate $x_{T}$ is fully determined and we only need that the $y$-coordinate of the mixed light equals a certain constant target value $y_{T}$. The light in the interval $[\theta, \theta+\mathrm{d} \theta]$ emitted from the TIR collimator is the sum of beams with intensity $\sigma_{i} \mathcal{I}\left(\eta_{i}(\theta)\right) \mathrm{d} \eta_{i}(\theta)$. The $y$-coordinate of this light is therefore

$$
y_{T}=\frac{\sum_{i=1}^{N} \sigma_{i} \mathcal{I}\left(\eta_{i}(\theta)\right) \mathrm{d} \eta_{i}(\theta)}{\sum_{i=1}^{N} \sigma_{i} \mathcal{I}\left(\eta_{i}(\theta)\right) / y\left(\eta_{i}(\theta)\right) \mathrm{d} \eta_{i}(\theta)} .
$$

Using (3) we find the following differential equation:

$$
\sum_{i=1}^{N} \sigma_{i} \frac{\mathcal{I}\left(\eta_{i}(\theta)\right)}{y\left(\eta_{i}(\theta)\right)} \eta_{i}^{\prime}(\theta)=c \mathcal{G}(\theta) / y_{T} .
$$


Figure 3 Geometry of the free surfaces B and C. The grey arrow shows the 'TIR route' of the light.

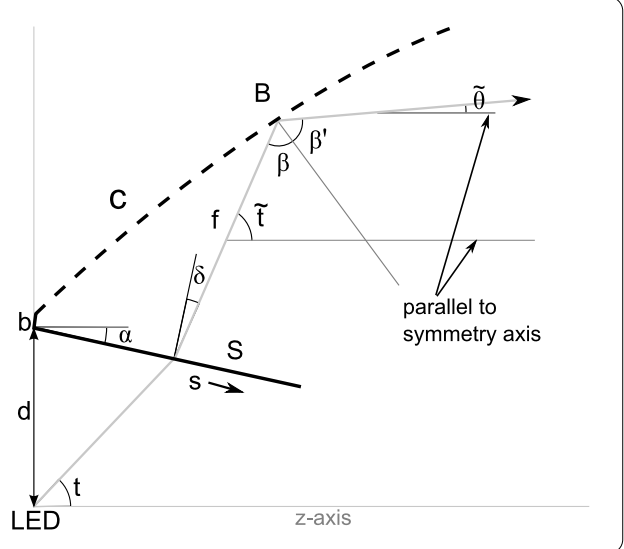

\subsection{Free surface calculations}

The light paths in the TIR collimator shown in Figure 1 correspond to three transfer functions, one transfer function for each of the free surfaces A, B or C. These free surfaces can be calculated from the transfer functions using the 'generalized functional method' developed by Bortz and Shatz $[10,11]$. They derived a differential equation that describes the location of a free surface, given a surface $S$ from which the rays depart with a given angle $\tilde{t}$ :

$$
\frac{\mathrm{d} f}{\mathrm{~d} s}=\frac{\mathrm{d} \tilde{t}}{\mathrm{~d} s} \tan (\beta) f+(\tan (\beta) \cos (\delta)+\sin (\delta))
$$

Here $f$ is the distance a light ray travels from the surface $S$ to the free surface, $s$ is the arclength along $S, \tilde{t}$ is the angle of the ray leaving $S$ with respect to the $z$-axis, and $\delta$ is the ray-emission angle measured counterclockwise with respect to the normal of $S$. The angle $\beta$ is the angle of incidence on the free surface with respect to the surface normal. For the reflective surfaces $B$ and $C$, the variables are illustrated in Figure 3. We like to formulate this differential equation in terms of $t$ instead of $s$. Multiplication by $\mathrm{d} s / \mathrm{d} t$ gives

$$
\frac{\mathrm{d} f}{\mathrm{~d} t}=\frac{\mathrm{d} \tilde{t}}{\mathrm{~d} t} \tan (\beta) f+\frac{\mathrm{d} s}{\mathrm{~d} t}(\tan (\beta) \cos (\delta)-\sin (\delta))
$$

The parameters $\beta$ and $\delta$ depend on $t$ and are derived below.

Light propagates through the collimator by two type of routes. In the 'TIR route', light is refracted by surface $S$, reflected by surface B or C by total internal reflection and finally refracted by surface $T$. In the 'lens route', light is refracted by surface A and subsequently refracted by surface $T$.

First consider the surfaces B and C. These surfaces are on the 'TIR route', which is shown in Figure 3. Surface B is bounded at one side by the rays that leave the source at angle $t=\tau_{1}$. The boundary between surface $B$ and $C$ is marked by the rays that leave the collimator at angle $\theta=0$, and we define the angle of this ray when leaving the light source to be $t=\tau_{2}$. The angles $\tau_{1}$ and $\tau_{2}$ are illustrated in Figure 1. First the light is refracted at surface $S$. Let $d$ be the distance from the left of surface $S$ to the LED and $\alpha$ the clockwise angle of this surface with respect to the symmetry-axis. A ray that leaves the LED at angle $t$, will hit 
surface $S$ at $\left(x_{S}(t), y_{S}(t)\right)$ and leave surface $S$ with angle $\tilde{t}(t)$ given by

$$
\begin{aligned}
& x_{S}(t)=\frac{d}{\tan (t)+\tan (\alpha)}, \\
& y_{S}(t)=\frac{d \tan (t)}{\tan (t)+\tan (\alpha)}, \\
& \tilde{t}(t)=\arccos \left(\frac{\cos (\alpha+t)}{n}\right)-\alpha .
\end{aligned}
$$

Relation (9c) was derived using Snell's law of refraction. The refractive index of the material of the collimator is denoted by $n$. From (9a) and (9b) we find $s$, which is defined to be 0 at $t=\pi / 2$. Also we calculate $\delta$ :

$$
\begin{aligned}
& s(t)=\frac{d}{\cos (\alpha) \tan (t)+\sin (\alpha)}, \\
& \delta(t)=\tilde{t}(t)+\alpha-\frac{\pi}{2} .
\end{aligned}
$$

Subsequently, the rays are reflected at surface B or C. For a reflective surface we have [10]

$$
\beta(t)=\frac{1}{2}(\tilde{t}(t)-\tilde{\theta}(t)-\pi)
$$

Here $\tilde{\theta}$ is the angle of the rays with respect to the $z$-axis after reflection. Before the rays leave the TIR collimator, they are once more refracted by surface $T$. Rays that leave the collimator at angle $\theta=\eta^{-1}(t)$ must enter surface $T$ at angle

$$
\tilde{\theta}(t)= \pm \arcsin \left(\sin \left(\eta^{-1}(t)\right) / n\right)
$$

where the sign is negative if the rays cross the $z$-axis, and positive otherwise. Equation (12) is derived using Snell's law. Now we can calculate $f(t)$ by numerically integrating the ODE (8) backwards, starting at $t=\pi / 2$. The parameters in (8) are given by (9c), (10a), (10b), (11) and (12). For surface $C$, a plus sign is chosen in (12) and for surface B a minus sign. The integration for surface $C$ starts with $f(\pi / 2)=b \geq 0$, which is usually chosen larger than 0 to prevent a sharp edge of the collimator for manufacturing purposes. At $t=\tau_{2}$, the final value $f\left(\tau_{2}\right)$ of the calculation of surface $C$ is chosen as starting value for the calculation of surface $\mathrm{B}$. The coordinates of the surfaces B and $\mathrm{C}$ can be calculated as

$$
\begin{aligned}
& x_{B / C}(t)=x_{S}(t)+f(t) \cos (\tilde{t}(t)), \\
& y_{B / C}(t)=y_{S}(t)+f(t) \sin (\tilde{t}(t)) .
\end{aligned}
$$

Now consider surface A, the 'lens route' of the collimator. The light incident on surface A comes from a single point, therefore the arc-length along the source surface is 0 , so we take $s(t)=0$. Furthermore, we have $\tilde{t}(t)=t$. For a refractive surface we need the following expression for $\beta$ [11]:

$$
\tan (\beta)=\frac{\sin (\tilde{\theta}-\tilde{t})}{1 / n-\cos (\tilde{\theta}-\tilde{t})}
$$


Using (12), the differential equation (8) is now

$$
\frac{\mathrm{d} f}{\mathrm{~d} t}=\left(\frac{\sin (\tilde{\theta}-t)}{1 / n-\cos (\tilde{\theta}-t)}\right) f,
$$

which we solve by numerically integrating backwards subject to the end condition

$$
f\left(\tau_{1}\right)=\sqrt{x_{S}\left(\tau_{1}\right)^{2}+y_{S}\left(\tau_{1}\right)^{2}}
$$

Surface A can be calculated according to

$$
\begin{aligned}
& x_{A}(t)=f(t) \cos (t), \\
& y_{A}(t)=f(t) \sin (t) .
\end{aligned}
$$

\section{A TIR collimator with three transfer functions}

Our goal is to design a TIR collimator that has a beam with a specified intensity output $\mathcal{G}(\theta)$ and uniform chromaticity coordinates $\left(x_{T}, y_{T}\right)$. To achieve this, the transfer functions must satisfy (3) and (6). The layout of the TIR collimator as shown in Figure 1 corresponds to three transfer functions, so $N=3$. For the lens part and surface $\mathrm{C}$, $t$ increases with $\theta$, so $\sigma_{1}=\sigma_{3}=1$. For surface B, $t$ decreases for increasing values of $\theta$, and thus $\sigma_{2}=-1$. We use the following convention: $\mathcal{I}_{i}(\theta)=\mathcal{I}\left(\eta_{i}(\theta)\right)$ and $y_{i}(\theta)=y\left(\eta_{i}(\theta)\right)$. We now have the following system of differential equations:

$$
\left(\begin{array}{ccc}
\mathcal{I}_{1}(\theta) & -\mathcal{I}_{2}(\theta) & \mathcal{I}_{3}(\theta) \\
\mathcal{I}_{1}(\theta) / y_{1}(\theta) & -\mathcal{I}_{2}(\theta) / y_{2}(\theta) & \mathcal{I}_{3}(\theta) / y_{3}(\theta)
\end{array}\right)\left(\begin{array}{l}
\eta_{1}^{\prime}(\theta) \\
\eta_{2}^{\prime}(\theta) \\
\eta_{3}^{\prime}(\theta)
\end{array}\right)=c \mathcal{G}(\theta)\left(\begin{array}{c}
1 \\
1 / y_{T}
\end{array}\right) .
$$

The initial and end conditions for the transfer functions follow from the signs $\sigma_{i}$ and the boundaries $\tau_{1}$ and $\tau_{2}$ between the segments:

$$
\begin{aligned}
& \eta_{1}(0)=0, \quad \eta_{2}(0)=\tau_{2}, \quad \eta_{3}(0)=\tau_{2}, \\
& \eta_{1}\left(\theta_{\max }\right)=\tau_{1}, \quad \eta_{2}\left(\theta_{\max }\right)=\tau_{1}, \quad \eta_{3}\left(\theta_{\max }\right)=\pi / 2 .
\end{aligned}
$$

The system (18) is underdetermined, therefore we add an extra equation. We choose an equation which is as simple as possible, has an obvious physical interpretation and yields a regular coefficient matrix for the ODE system. The equation we choose corresponds to the requirement that the intensity resulting from one of the transfer functions contributes a factor $r \in(0,1)$ to the total target intensity. Let $j$ be the index of this transfer function, then we impose

$$
\sigma_{j} \mathcal{I}_{j}(\theta) \eta_{j}^{\prime}(\theta)=r c \mathcal{G}(\theta)
$$

For $j=1$, the coefficient matrix of the system is singular for $\theta=0$, and for $j=3$, the coefficient matrix is singular for $\theta=\theta_{\max }$. Such a singular coefficient matrix does not occur for 
$j=2$, so this will be our choice. The ODE system is now

$$
\left(\begin{array}{ccc}
\mathcal{I}_{1}(\theta) & -\mathcal{I}_{2}(\theta) & \mathcal{I}_{3}(\theta) \\
\mathcal{I}_{1}(\theta) / y_{1}(\theta) & -\mathcal{I}_{2}(\theta) / y_{2}(\theta) & \mathcal{I}_{3}(\theta) / y_{3}(\theta) \\
0 & -\mathcal{I}_{2}(\theta) & 0
\end{array}\right)\left(\begin{array}{l}
\eta_{1}^{\prime}(\theta) \\
\eta_{2}^{\prime}(\theta) \\
\eta_{3}^{\prime}(\theta)
\end{array}\right)=c \mathcal{G}(\theta)\left(\begin{array}{c}
1 \\
1 / y_{T} \\
r
\end{array}\right) .
$$

The system can be inverted, yielding the following explicit system

$$
\begin{aligned}
& \eta_{1}^{\prime}(\theta)=\frac{c \mathcal{G}(\theta)}{\mathcal{I}_{1}(\theta)} \frac{y_{1}(\theta)}{y_{1}(\theta)-y_{3}(\theta)}\left(1-r-\frac{y_{3}(\theta)}{y_{T}}+r \frac{y_{3}(\theta)}{y_{2}(\theta)}\right), \\
& \eta_{2}^{\prime}(\theta)=-r \frac{c \mathcal{G}(\theta)}{\mathcal{I}_{2}(\theta)}, \\
& \eta_{3}^{\prime}(\theta)=\frac{c \mathcal{G}(\theta)}{\mathcal{I}_{3}(\theta)} \frac{y_{3}(\theta)}{y_{3}(\theta)-y_{1}(\theta)}\left(1-r-\frac{y_{1}(\theta)}{y_{T}}+r \frac{y_{1}(\theta)}{y_{2}(\theta)}\right) .
\end{aligned}
$$

The system (22a)-(22c) with boundary conditions (19a) and (19b) has three unknown functions $\eta_{1}(\theta), \eta_{2}(\theta)$ and $\eta_{3}(\theta)$. The functions $\mathcal{I}(t)$ and $y(t)$ are known from measurements on the LED. The function $\mathcal{G}(\theta)$ can be chosen by the optical designer as a finite function on $\left[0, \theta_{\max }\right]$. The constants $c, y_{T}$ and $r$ cannot be chosen freely, we will show that their values follow from conservation of luminous flux, the law of color mixing and the choice of $\tau_{1}$ and $\tau_{2}$, respectively. Also the values of $\tau_{1}$ and $\tau_{2}$ cannot be chosen freely, we will derive an inequality that guarantees monotonicity of the transfer functions.

Equation (22a) has a removable singularity at $\theta=0$, because $\mathcal{G}(0)=0$ and the initial values of the transfer functions imply $\eta_{1}(0)=0$ and thus $\mathcal{I}_{1}(0)=\mathcal{I}(0)=0$. We calculate $\eta_{1}^{\prime}(0)$ using l'Hôpital's rule:

$$
\eta_{1}^{\prime}(0)=\sqrt{\frac{c \mathcal{G}_{+}^{\prime}(0)}{\mathcal{I}_{+}^{\prime}(0)} \frac{y_{1}(0)}{y_{1}(0)-y_{3}(0)}\left(1-\frac{y_{3}(0)}{y_{T}}\right)} .
$$

We choose the positive sign in front of the square root since $\eta_{1}^{\prime}(0)$ should be positive. Here $\mathcal{G}_{+}^{\prime}(0)$ and $\mathcal{I}_{+}^{\prime}(0)$ are the right derivatives of $\mathcal{G}(\theta)$ at $\theta=0$ and of $\mathcal{I}(t)$ at $t=0$, respectively. These right derivatives are positive because $\mathcal{I}(t)$ and $\mathcal{G}(\theta)$ are positive at $t>0$ and $\theta>0$. We have $y(t)>0$ by definition of chromaticity coordinates, and we assume based on measurements that $y\left(\tau_{1}\right)<y\left(\tau_{2}\right)$ and thus $y_{1}(0)<y_{3}(0)$. From this we see that we need to choose $\tau_{2}$ such that $y\left(\tau_{2}\right)>y_{T}$, so the right hand side of (23) is positive and real.

\subsection{The values of $c, y_{T}$ and $r$}

The system (21) with boundary conditions (19a) and (19b) appears to be overdetermined. However, the system contains three unknown parameters which still need to be chosen. We derive values for three constants $c, y_{T}$ and $r$ given the boundary conditions and assuming monotonicity of the transfer functions. Later we show that our choice of the constants $c, y_{T}$ and $r$ imply that three of the boundary conditions are superfluous.

The first unknown value is the constant $c$. Integration of the first row of (21), using the given boundary conditions and $\mathcal{I}_{i}(\theta)=\mathcal{I}\left(\eta_{i}(\theta)\right)$, yields

$$
c \int_{0}^{\theta_{\max }} \mathcal{G}(\theta) \mathrm{d} \theta=\int_{0}^{\theta_{\max }} \sum_{i=1}^{3} \sigma_{i} \mathcal{I}_{i}(\theta) \eta_{i}(\theta) \mathrm{d} \theta=\int_{0}^{\pi / 2} \mathcal{I}(t) \mathrm{d} t
$$


The function $\mathcal{I}(t)$ is known from measurements on the LED, the function $\mathcal{G}(\theta)$ is chosen by the optical designer, so from this relation we derive the value of the constant $c$. This relation corresponds to conservation of luminous flux (equation (2)).

The second unknown is the target chromaticity value $y_{T}$. Integration of the second row of (21) using the given boundary conditions and substitution of (24) yields

$$
1 / y_{T} \int_{0}^{\pi / 2} \mathcal{I}(t) \mathrm{d} t=\int_{0}^{\pi / 2} \mathcal{I}(t) / y(t) \mathrm{d} t
$$

This relation shows that $y_{T}$ is the weighted harmonic average of the $y$-chromaticity coordinate of the light source. Like $\mathcal{I}(t)$, the function $y(t)$ is known from measurements on the LED, thus we can derive the value of $y_{T}$.

The third unknown is $r$. Integration of the third row of (21) with the given boundary conditions and substitution of (24) yields

$$
r \int_{0}^{\pi / 2} \mathcal{I}(t) \mathrm{d} t=\int_{\tau_{1}}^{\tau_{2}} \mathcal{I}(t) \mathrm{d} t
$$

This relations corresponds to conservation of luminous flux for the second transfer function.

\subsection{Monotonicity of the transfer functions}

The transfer functions calculated from (22a)-(22c) should be monotonic, otherwise they have no physical meaning. From (22b) we can easily see that $\eta_{2}^{\prime}(\theta) \leq 0$ because $r>0$, $\mathcal{G}(\theta) \geq 0$ and $\mathcal{I}(t) \geq 0$, thus, $\eta_{2}(\theta)$ is monotonically decreasing. The monotonicity of $\eta_{1}$ and $\eta_{3}$ is more complicated to show and we need some additional assumptions to derive a sufficient condition for monotonicity.

Theorem 1 Assume that the chromaticity coordinate function $y(t)$ satisfies the inequalities

$$
\begin{aligned}
0<y\left(t_{1}\right)<y\left(\tau_{1}\right)<y\left(t_{2}\right)<y\left(\tau_{2}\right)<y\left(t_{3}\right) \\
\quad \forall t_{1}, t_{2}, t_{3} \text { s.t. } 0<t_{1}<\tau_{1}<t_{2}<\tau_{2}<t_{3}<\pi / 2,
\end{aligned}
$$

and the transfer functions satisfy the bounds

$$
0 \leq \eta_{1}(\theta) \leq \tau_{1}, \quad \tau_{1} \leq \eta_{2}(\theta) \leq \tau_{2}, \quad \tau_{2} \leq \eta_{3}(\theta) \leq \pi / 2 .
$$

If

$$
\frac{\int_{\tau_{1}}^{\tau_{2}} \mathcal{I}(t) \mathrm{d} t}{\int_{0}^{\pi / 2} \mathcal{I}(t) \mathrm{d} t}=r<\min \left(\frac{y\left(\tau_{1}\right)}{y_{T}}, \frac{y\left(\tau_{2}\right) / y_{T}-1}{y\left(\tau_{2}\right) / y\left(\tau_{1}\right)-1}, \frac{1-y\left(\tau_{1}\right) / y_{T}}{1-y\left(\tau_{1}\right) / y\left(\tau_{2}\right)}\right),
$$

then $\eta_{1}(\theta)$ and $\eta_{3}(\theta)$ are monotonically increasing.

Proof We need to prove that the derivatives of $\eta_{1}$ and $\eta_{3}$ are positive. From (22a), using assumptions (27) and (28), we find that $\eta_{1}$ is monotonically increasing if

$$
1-r-\frac{y_{3}(\theta)}{y_{T}}+r \frac{y_{3}(\theta)}{y_{2}(\theta)} \leq 0
$$


and likewise $\eta_{3}$ is monotonically increasing if

$$
1-r-\frac{y_{1}(\theta)}{y_{T}}+r \frac{y_{1}(\theta)}{y_{2}(\theta)} \geq 0 .
$$

Subtracting the second inequality from the first we obtain

$$
t-\frac{1}{y_{T}}\left(y_{3}(\theta)-y_{1}(\theta)\right)+\frac{r}{y_{2}(\theta)}\left(y_{3}(\theta)-y_{1}(\theta)\right) \leq 0 .
$$

Using (27) and (28) we find from the assumption $r<y\left(\tau_{1}\right) / y_{T}$ that

$$
\frac{r}{y_{2}(\theta)} \leq \frac{1}{y_{T}}
$$

Define

$$
M(\theta)=\frac{1-r}{1 / y_{T}-r / y_{2}(\theta)} .
$$

Using $\frac{r}{y_{2}(\theta)} \leq \frac{1}{y_{T}}$, the inequality for the monotonicity of the first transfer function can be rewritten as $M(\theta) \leq y_{3}(\theta)$ and the inequality for the third transfer function as $y_{1}(\theta) \leq M(\theta)$. We combine these two results to obtain

$$
y_{1}(\theta) \leq M(\theta) \leq y_{3}(\theta)
$$

The function $M(\theta)$ it is monotonically increasing because $\eta_{2}(\theta)$ is monotonically decreasing. Therefore, if $y\left(\tau_{1}\right) \leq M(0)$, we find using (27) and (28) that $y_{1}(\theta) \leq M(\theta)$ for all $\theta$. Similarly, if $M\left(\theta_{\max }\right) \leq y\left(\tau_{2}\right)$, then $M(\theta) \leq y_{3}(\theta)$ for all $\theta$. The inequalities $y\left(\tau_{1}\right) \leq M(0)$ and $M\left(\theta_{\max }\right) \leq y\left(\tau_{2}\right)$ are equivalent to the second and third inequality in (29).

Figure 4 shows a scatter plot of values of $\tau_{1}$ and $\tau_{2}$ for which (29) is satisfied for an LED which was also used in the numerical experiments. The acceptable values of $\tau_{1}$ and $\tau_{2}$ are bounded by the lines $\tau_{1}=t_{\mathrm{av}}$ and $\tau_{2}=t_{\mathrm{av}}$, where $t_{\mathrm{av}}$ is the such that $y\left(t_{\mathrm{av}}\right)=y_{T}$. In this case, the value of $t_{\mathrm{av}}$ is unique. From (29) we see that $\tau_{1}>t_{\mathrm{av}}$ results in $y\left(\tau_{1}\right)>y_{T}$, and thus $r<0$. Therefore we cannot guarantee the monotonicity of the transfer functions. Using (22c) we can verify that indeed the third transfer function is not monotonic at $\theta=\theta_{\max }$. Similarly, $\tau_{2}<t_{\mathrm{av}}$ results in $r<0$, and we can verify using (23) that the first transfer function is not monotonic at $\theta=0$.

\subsection{The initial value problem}

The ODE-system (21) with the boundary conditions (19a) and (19b) can be solved as an initial value problem. We remark that solving the system as an end value problem has no advantages or disadvantages. We discard the end conditions and solve the initial value problem using a Runge-Kutta method. The end conditions are satisfied as a result of our choices of $c, y_{T}$ and $r$.

Theorem 2 Assume monotonicity of the transfer functions. The solution of the initial value problem defined by the ODE system (21) and the initial conditions $\eta_{1}(0)=0, \eta_{2}(0)=\eta_{3}(0)=$ $\tau_{2}$ satisfies the end conditions $\eta_{1}\left(\theta_{\max }\right)=\eta_{2}\left(\theta_{\max }\right)=\tau_{1}, \eta_{3}\left(\theta_{\max }\right)=\pi / 2$. 


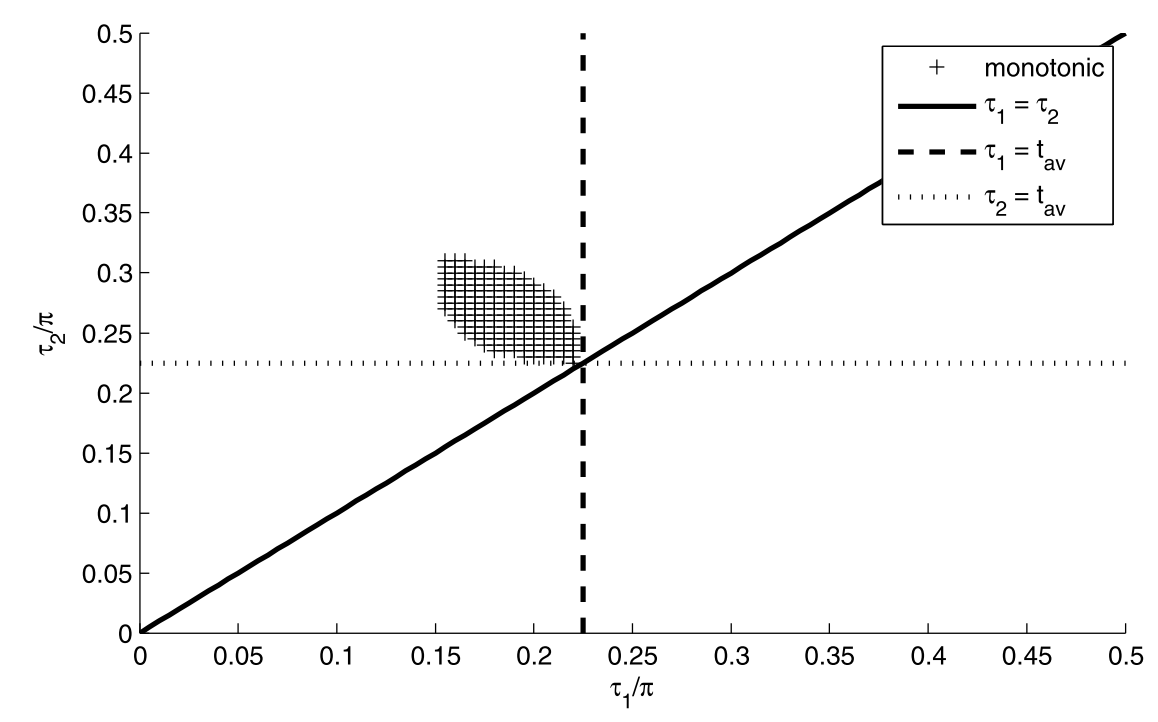

Figure 4 Scatter plot of the values of $\tau_{1}$ and $\tau_{2}$ for LED16 that guarantee monotonic transfer functions. $t_{\mathrm{av}}$ is such that $y\left(t_{\mathrm{av}}\right)=y_{T}$.

Proof First we show that $\eta_{2}\left(\theta_{\max }\right)=\tau_{1}$ is satisfied. Integration of the last row of (21) from $\theta=0$ to $\theta=\theta_{\max }$ using (26) gives

$$
-\int_{0}^{\theta_{\max }} \mathcal{I}_{2}(\theta) \eta_{2}^{\prime}(\theta) \mathrm{d} \theta=-\int_{\tau_{2}}^{\eta_{2}\left(\theta_{\max }\right)} \mathcal{I}(t) \mathrm{d} t=r c \int_{0}^{\theta_{\max }} \mathcal{G}(\theta) \mathrm{d} \theta=\int_{\tau_{1}}^{\tau_{2}} \mathcal{I}(t) \mathrm{d} t .
$$

So we find

$$
\int_{\tau_{2}}^{\eta_{2}\left(\theta_{\max }\right)} \mathcal{I}(t) \mathrm{d} t+\int_{\tau_{1}}^{\tau_{2}} \mathcal{I}(t) \mathrm{d} t=0
$$

Because $\mathcal{I}(t)>0$ for all $t$ except for two points at the boundary, we can conclude $\eta_{2}(\theta)=\tau_{1}$. Note that this implies $\tau_{1} \leq \eta_{2}(\theta) \leq \tau_{2}$.

Using the monotonicity of the transfer functions, we can integrate the left hand side of the first row of (21):

$$
\begin{aligned}
& \int_{0}^{\theta_{\max }}\left(\mathcal{I}_{1}(\theta) \eta_{1}^{\prime}(\theta)-\mathcal{I}_{2}(\theta) \eta_{2}^{\prime}(\theta)+\mathcal{I}_{3}(\theta) \eta_{3}^{\prime}(\theta)\right) \mathrm{d} \theta \\
& \quad=\int_{0}^{\eta_{1}\left(\theta_{\max }\right)} \mathcal{I}(t) \mathrm{d} t-\int_{\tau_{2}}^{\tau_{1}} \mathcal{I}(t) \mathrm{d} t+\int_{\tau_{2}}^{\eta_{3}\left(\theta_{\max }\right)} \mathcal{I}(t) \mathrm{d} t .
\end{aligned}
$$

For the right hand side we have due to (24)

$$
c \int_{0}^{\theta_{\max }} \mathcal{G}(\theta) \mathrm{d} \theta=\int_{0}^{\pi / 2} \mathcal{I}(t) \mathrm{d} t
$$

By subtracting the last two relations we find

$$
\int_{\tau_{1}}^{\eta_{1}\left(\theta_{\max }\right)} \mathcal{I}(t) \mathrm{d} t+\int_{\pi / 2}^{\eta_{3}\left(\theta_{\max }\right)} \mathcal{I}(t) \mathrm{d} t=0
$$


Similarly, we can derive from the second row of (21) using equation (25)

$$
\int_{\tau_{1}}^{\eta_{1}\left(\theta_{\max }\right)} \frac{\mathcal{I}(t)}{y(t)} \mathrm{d} t+\int_{\pi / 2}^{\eta_{3}\left(\theta_{\max }\right)} \frac{\mathcal{I}(t)}{y(t)} \mathrm{d} t=0
$$

The functions $\mathcal{I}(t)$ and $1 / y(t)$ are continuous, and $\mathcal{I}(t)$ does not change sign in the interval $(0, \pi / 2)$. Using the expanded first mean-value theorem for integrals [12, p.487], we find that for some $t_{1} \in\left(\eta_{1}\left(\theta_{\max }\right), \tau_{1}\right)$ and some $t_{3} \in\left(\eta_{2}\left(\theta_{\max }\right), \pi / 2\right)$ we have

$$
\frac{1}{y\left(t_{1}\right)} \int_{\tau_{1}}^{\eta_{1}\left(\theta_{\max }\right)} \mathcal{I}(t) \mathrm{d} t+\frac{1}{y\left(t_{3}\right)} \int_{\pi / 2}^{\eta_{3}\left(\theta_{\max }\right)} \mathcal{I}(t) \mathrm{d} t=0
$$

The equations (*) and (**) form a linear system for the integrals of $\mathcal{I}(t)$ over $\left[\tau_{1}, \eta_{1}\left(\theta_{\max }\right)\right]$ and $\left[\pi / 2, \eta_{3}\left(\theta_{\max }\right)\right]$. If $t_{1}<\tau_{2}$, we find from the assumption $y\left(t_{1}\right)<y\left(t_{3}\right)$ that

$$
\operatorname{det}\left(\begin{array}{cc}
1 & 1 \\
1 / y\left(t_{1}\right) & 1 / y\left(t_{3}\right)
\end{array}\right) \neq 0,
$$

and thus

$$
\begin{aligned}
& \int_{\tau_{1}}^{\eta_{1}\left(\theta_{\max }\right)} \mathcal{I}(t) \mathrm{d} t=0, \\
& \int_{\pi / 2}^{\eta_{3}\left(\theta_{\max }\right)} \mathcal{I}(t) \mathrm{d} t=0 .
\end{aligned}
$$

Because $\mathcal{I}(t)>0$ for all $t$ except at the boundary points, we conclude

$$
\begin{aligned}
& \eta_{1}\left(\theta_{\max }\right)=\tau_{1}, \\
& \eta_{3}\left(\theta_{\max }\right)=\pi / 2 .
\end{aligned}
$$

\section{Numerical procedure and results}

We solved the mathematical model described in the previous section to design three different TIR collimators. The collimators were designed for two different LEDs, which we refer to as LED16 and LED02. Both of them are Luxeon Rebel IES white LEDs without a dichroic coating, and have a larger than usual CoA variation. The intensity and chromaticity-coordinates of the LEDs were measured, and the measured data were interpolated. The interpolation polynomials have been used to approximate $\mathcal{I}(t)$ and $y(t)$ in (22a)-(22c). The first two collimators were designed for LED16 and have a Gaussianshaped target intensity profile. The two collimators differ in their values for $\tau_{1}$ and $\tau_{2}$. The third collimator was designed for LED02 and has a block-shaped target intensity profile. The collimators were evaluated using the LightTools software package [13].

\subsection{Modelling of the LEDs}

The LEDs were measured using a goniophotometer [14]. A goniophotometer is a device that measures intensity, chromaticity coordinates and many other characteristics of light at different solid angles. Our LEDs were measured at 46 different angles $t$ and 4 different angles $u$. For each LED, the chromaticity values were averaged and the intensities were 
Table 1 Coefficients from the linear least squares fits

\begin{tabular}{lllrrrrrr}
\hline LED & $\boldsymbol{i}$ & $\mathbf{0}$ & \multicolumn{1}{l}{$\mathbf{2}$} & $\mathbf{3}$ & \multicolumn{1}{c}{$\mathbf{4}$} & $\mathbf{5}$ & $\mathbf{6}$ & \multicolumn{1}{c}{$\mathbf{7}$} \\
\hline 16 & $C_{i}$ & 0 & -181.8279 & 76.0797 & 221.9411 & -624.0461 & 499.8253 & -127.0787 \\
& $D_{i}^{x}$ & 0.4013 & 0.0578 & -0.0367 & 0.0271 & -0.0564 & 0.0444 & -0.0116 \\
& $D_{i}^{y}$ & 0.3546 & 0.0988 & -0.0557 & 0.0255 & -0.0371 & 0.0196 & -0.0034 \\
02 & $C_{i}$ & 0 & -148.8533 & -60.3797 & 520.5934 & -913.6760 & 636.8623 & -153.5863 \\
& $D_{i}^{x}$ & 0.4591 & 0.0452 & -0.0590 & 0.0916 & -0.1399 & 0.1030 & -0.0271 \\
& $D_{i}^{y}$ & 0.3792 & 0.0892 & -0.1790 & 0.3596 & -0.4585 & 0.2823 & -0.0658 \\
\hline
\end{tabular}

summed over the angle $u$. These data have been interpolated with a least squares fit using the following polynomials:

$$
\begin{aligned}
& I(t)=\sum_{i=2}^{7} C_{i}\left(t^{i}-(\pi / 2)^{i}\right), \\
& x(t)=D_{0}^{x}+\sum_{i=2}^{7} D_{i}^{x} t^{i}, \\
& y(t)=D_{0}^{y}+\sum_{i=2}^{7} D_{i}^{y} t^{i} .
\end{aligned}
$$

The polynomial for the intensity was chosen because it equals 0 at $t=\pi / 2$ and has zero derivative at $t=0$, both properties are characteristic for the intensity distribution of an LED. The effective intensity equals $\mathcal{I}(t)=\sin (t) I(t)$. The polynomials for the chromaticity coordinates were chosen because their derivative equals 0 at $t=0$. The coefficients for the two LEDs can be found in Table 1.

In the LightTools software package, two three-dimensional models were built to simulate the LEDs. The range $\left(0, \frac{\pi}{2}\right)$ of the angle $t$ was discretized into 46 different subintervals, labeled $k=1,2, \ldots, 46$. For $k=1$ we have the interval $\left(0, \frac{\pi}{180}\right)$, for $k=2, \ldots, 45$ we have $\left(\frac{(2 k-3) \pi}{180}, \frac{(2 k-1) \pi}{180}\right)$ and finally for $k=46$ we have $\left(\frac{89 \pi}{180}, \frac{\pi}{2}\right)$. The intensity and chromaticity coordinates of the LED models in these subintervals correspond to the measured data of the real LEDs at the angles $t=\frac{(2 k-2) \pi}{180}$. The size of the LED model was reduced to $0.01 \mathrm{~mm}$ by $0.01 \mathrm{~mm}$ to simulate a point light source. A comparison of the measured data, the least squares fit and the raytracing results of the LightTools model of LED16 without collimator can be seen in Figures 5 and 6. A scatter plot of the measured $x$ and $y$ chromaticity coordinates for this LED was shown earlier in Figure 2. The plot shows the near-linear relationship between $x$ and $y$, indeed.

\subsection{Computation of the transfer functions}

Three example collimators have been calculated. The first collimator was designed for a Gaussian target intensity [15] with full width at half maximum (FWHM) [16] at $\pi / 9$. This yields the following effective target intensity:

$$
\mathcal{G}(\theta)=\sin (\theta) \exp \left(-4 \ln (2)\left(\frac{\theta}{\theta_{\text {FWHM }}}\right)^{2}\right)
$$

with $0 \leq \theta \leq 1.25 \theta_{\mathrm{FWHM}}=\theta_{\mathrm{max}}, \theta_{\mathrm{FWHM}}=\pi / 9$. The collimator was designed for LED16. The choice of $\tau_{1}$ and $\tau_{2}$ is restricted by (29). This relation is highly nonlinear. A scatter plot of values of $\tau_{1}$ and $\tau_{2}$ that satisfy (29) for LED16 is shown in Figure 4. 


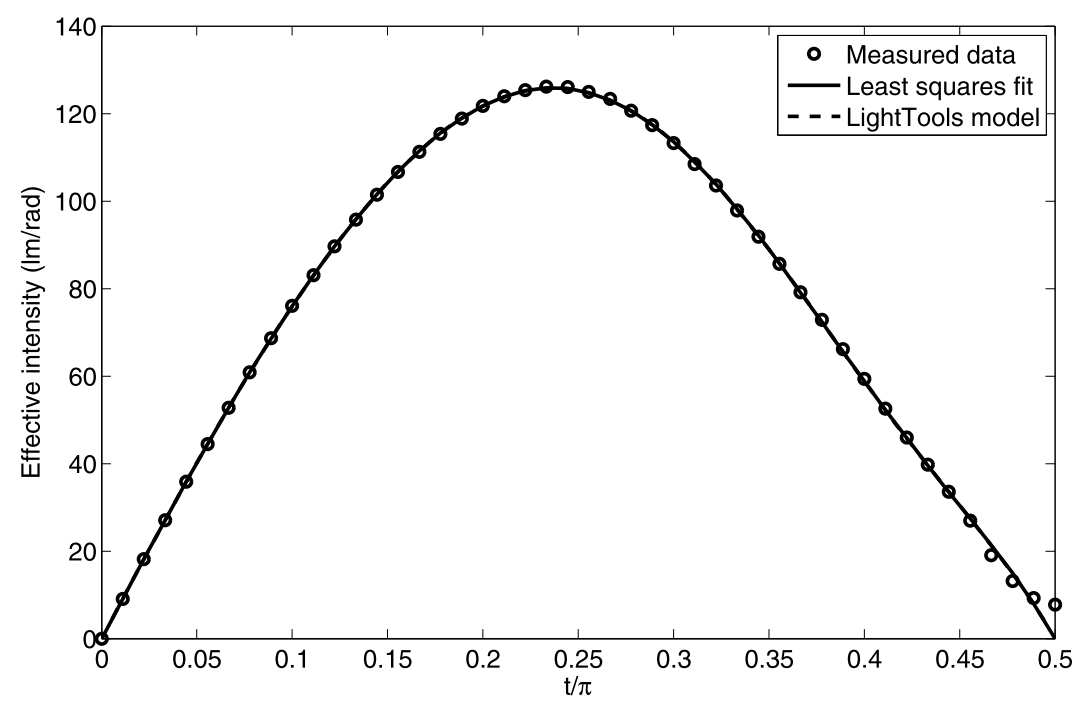

Figure 5 Comparison of the measured effective intensity $\mathcal{I}$, the least squares fit and the LightTools model of LED16. The graph of the LightTools model is not visible because it is hidden behind the least squares fit.

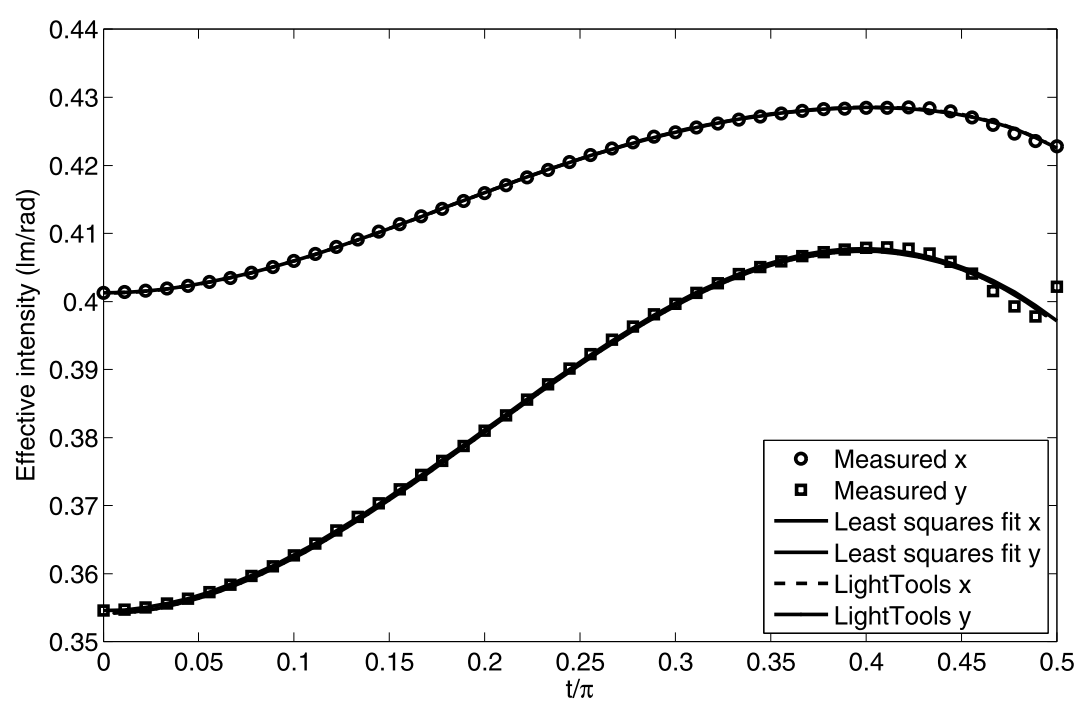

Figure 6 Comparison of measured chromaticity coordinates $x$ and $y$, the least squares fit and the LightTools Model of LED16. The graph of the LightTools model is not visible because it is hidden behind the least squares fit.

We chose $\tau_{1}=0.2 \pi$ and $\tau_{2}=0.25 \pi$. The second collimator was designed for the same LED and target intensity, but this time we chose $\tau_{1}=0.16 \pi$ and $\tau_{2}=0.3 \pi$, which gives a larger second segment. The third collimator was designed for LED02. The target intensity was chosen to be a block function, yielding the effective intensity $\mathcal{G}(\theta)=\sin (\theta)$, with $0 \leq$ $\theta \leq \theta_{\max }=\pi / 9$. We chose $\tau_{1}=0.2 \pi$ and $\tau_{2}=0.25 \pi$, which satisfies (29). An overview of the values chosen and calculated for the three collimators is shown in Table 2.

The ODE system (22a)-(22c) with initial conditions (19a) was solved using the ODEsolver ode 45 in Matlab. The calculation times were a few seconds on a laptop computer 
Table 2 Parameter values and characteristics for the three different collimators

\begin{tabular}{llllllll}
\hline Collimator & LED & $\boldsymbol{\tau}_{\mathbf{1}}$ & $\boldsymbol{\tau}_{\mathbf{2}}$ & $\boldsymbol{r}$ & $\boldsymbol{y}_{\boldsymbol{T}}$ & $\boldsymbol{c}$ & $\boldsymbol{\theta}_{\max }$ \\
\hline Gaussian, small 2nd segment & LED16 & $0.20 \pi$ & $0.25 \pi$ & 0.1630 & 0.3862 & $5,585.9$ & $11.25 \pi$ \\
Gaussian, large 2nd segment & LED16 & $0.16 \pi$ & $0.30 \pi$ & 0.4417 & 0.3862 & $5,585.9$ & $11.25 \pi$ \\
Block profile & LED02 & $0.20 \pi$ & $0.25 \pi$ & 0.1612 & 0.3986 & $4,843.3$ & $9 \pi$ \\
\hline
\end{tabular}

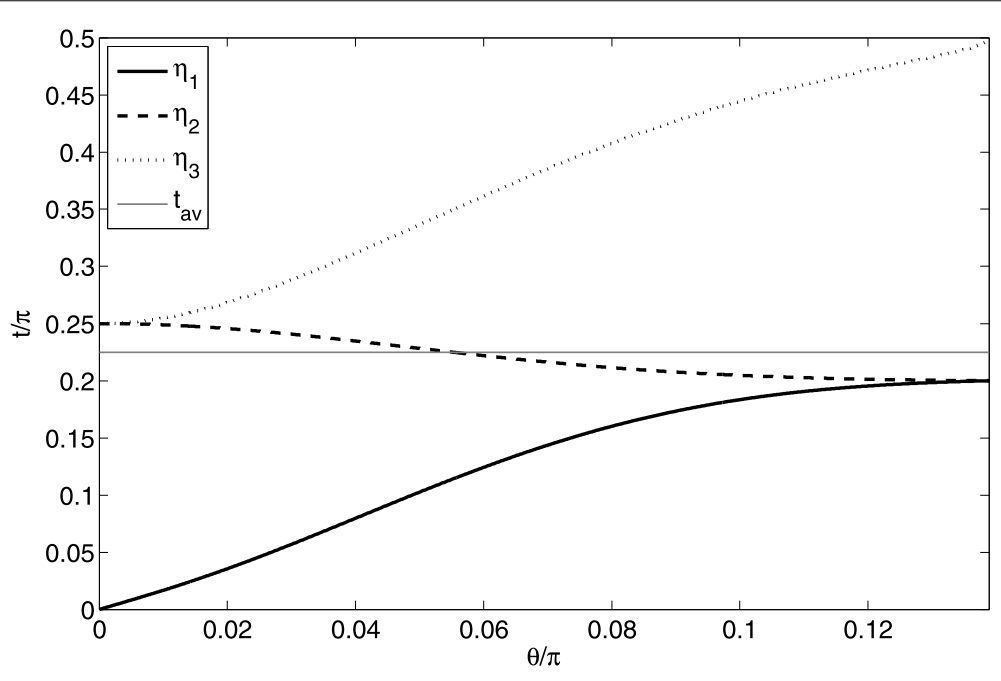

Figure 7 Transfer functions for the collimator with Gaussian profile and a small second segment.

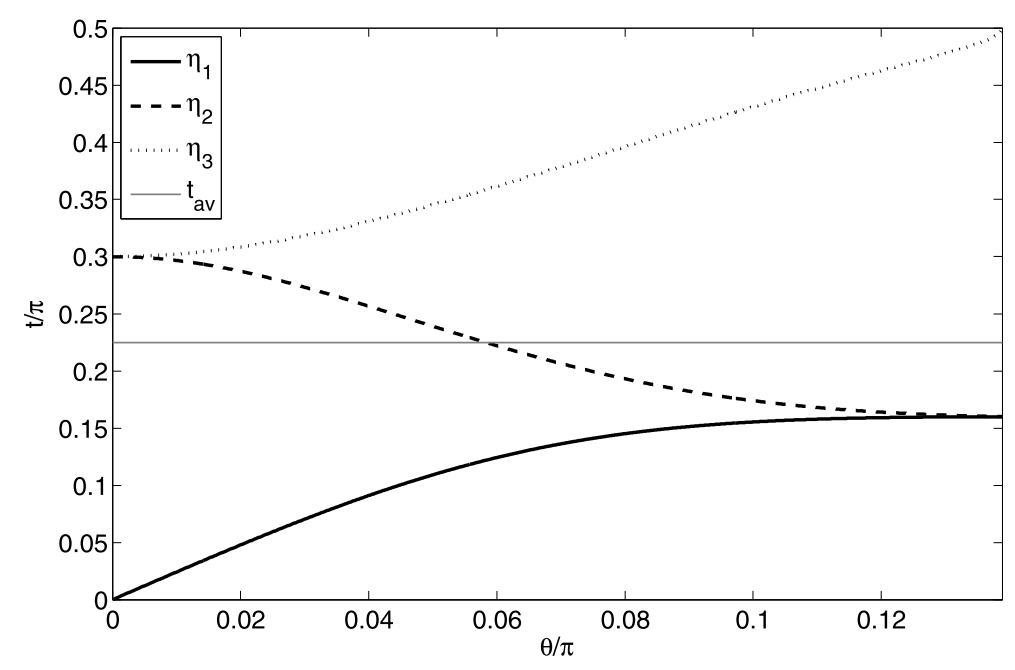

Figure 8 Transfer functions for the collimator with Gaussian profile and a large second segment.

with a $2.4 \mathrm{GHz}$ processor and $4 \mathrm{~GB}$ RAM. The calculated transfer functions are shown in Figure 7, 8 and 9. The transfer functions are indeed monotonic, as expected. Also, $\eta_{1}\left(\theta_{\max }\right)=\eta_{2}\left(\theta_{\max }\right)=\tau_{1}$ and $\eta_{3}\left(\theta_{\max }\right)=\pi / 2$, as anticipated.

\subsection{Performance of the TIR collimators}

Subsequently, a TIR collimator was designed for each set of transfer functions, and evaluated using LightTools. We chose for all the collimators $d=4 \mathrm{~mm}, b=0.4 \mathrm{~mm}$ and 


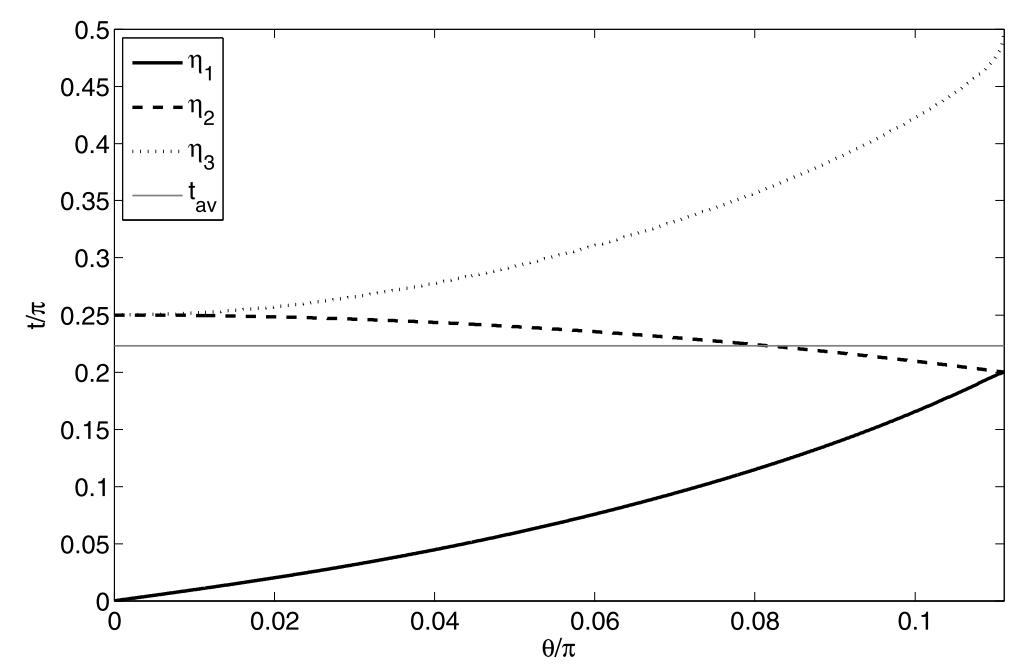

Figure 9 Transfer functions for the collimator with block profile.

Figure 10 LightTools model of the first collimator.

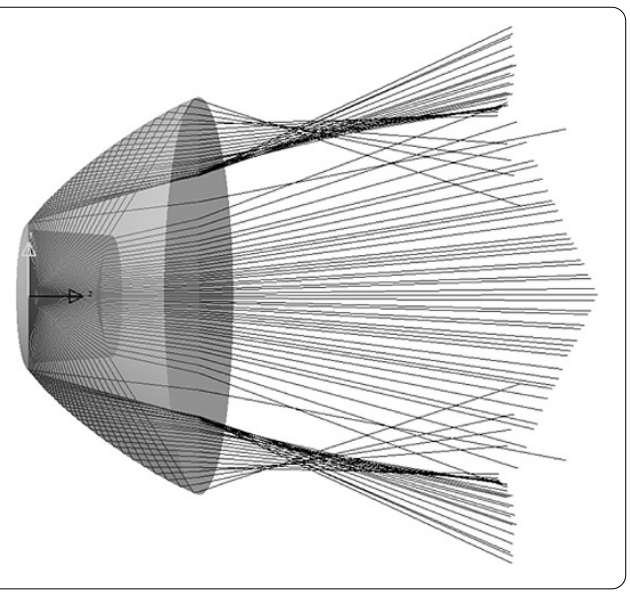

$\alpha=4 \pi / 180$. For every collimator, each free surface was discretized using 500 points and converted into a LightTools model. A screenshot of the LightTools model of the first collimator can be seen in Figure 10. Results of the simulations can be seen in Figures 11, 12 and 13. In these figures, we see the expected profiles of the effective intensity and chromaticity. Figures 11 and 12 look very similar, because the first and second collimator were designed with the same output specifications. In these figures, an irregularity is visible in the chromaticity coordinates near $\theta=\theta_{\max }$. This can be explained as follows. Every bar in the graph corresponds to a range of one degree $(\pi / 180 \mathrm{rad})$. We chose $\theta_{\max }=25 \pi / 180$, and thus the flux at this angle should be zero. Due to small errors in the free surfaces, a small number of rays exits the collimator at angles larger than $\theta_{\max }$. This happens at surface $C$, and therefore the chromaticity coordinates at $\theta>\theta_{\max }$ are larger than the target values. Because the luminous flux of this light is very small, the irregularity is not visible. A similar irregularity is visible for the collimator with the block profile, only with a smaller chromaticity difference.

Apart from this small irregularity, the variation in chromaticity is very small. The maximum difference between the average chromaticity of the LEDs and chromaticity coordi- 


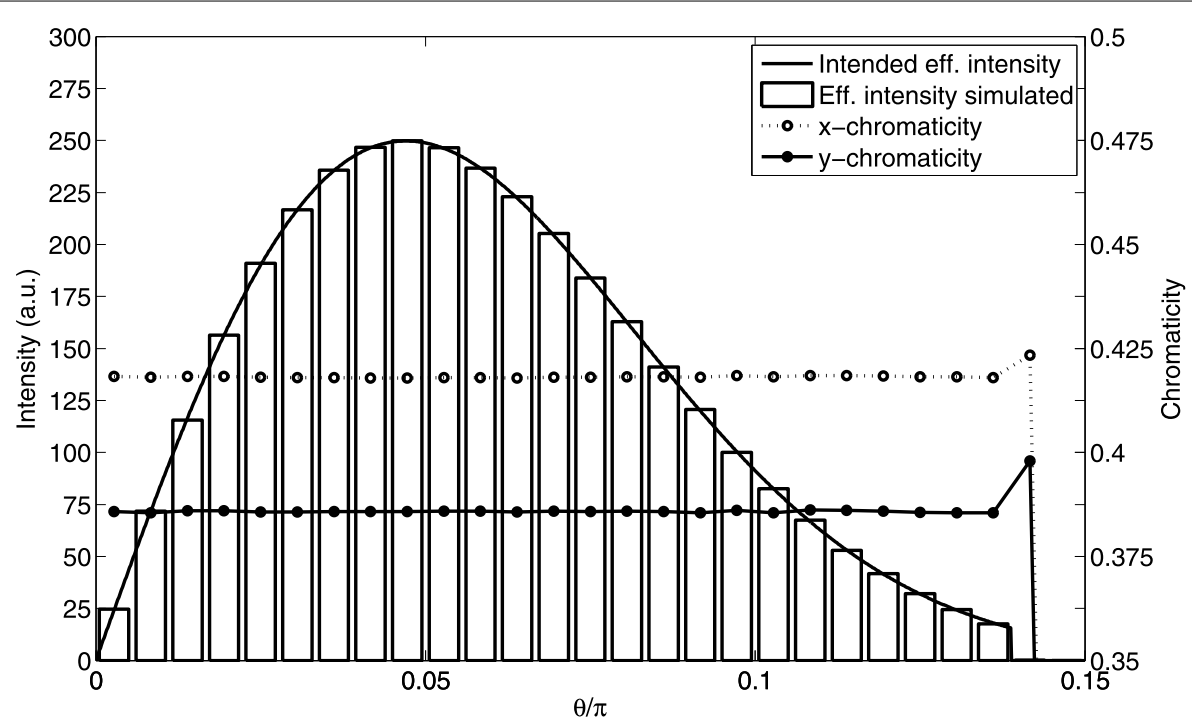

Figure 11 LightTools simulation results for the collimator with Gaussian profile and a small second segment.

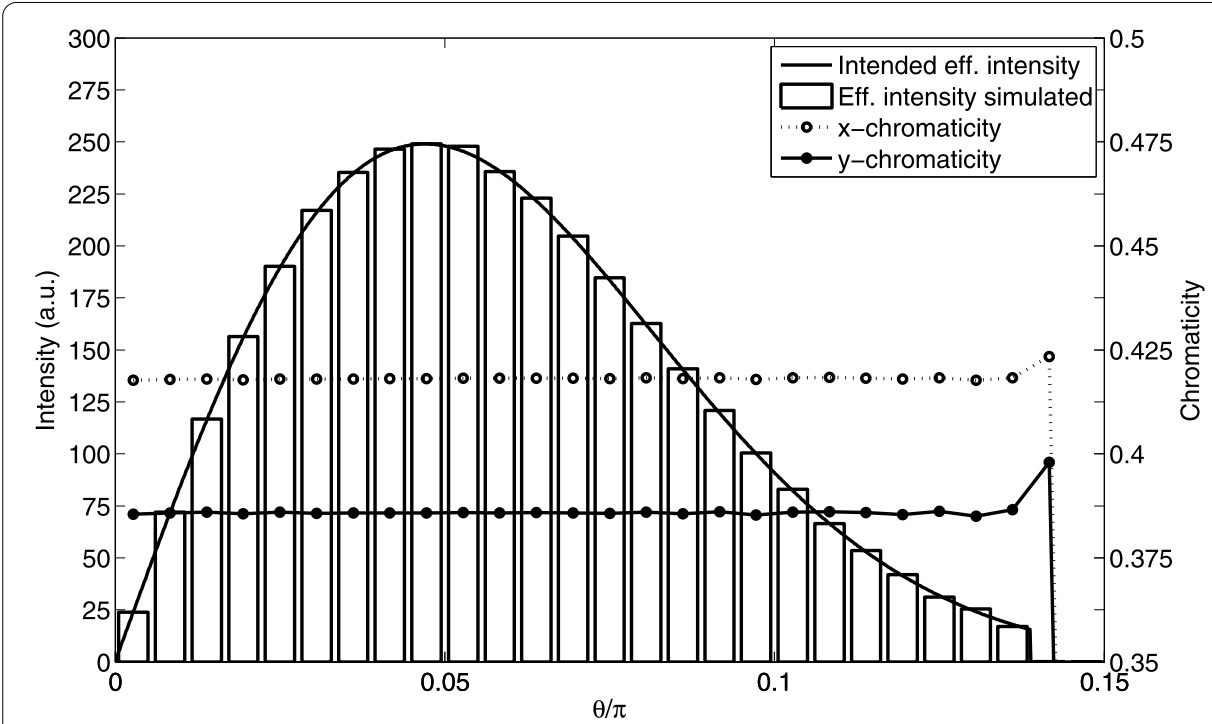

Figure 12 LightTools simulation results for the collimator with Gaussian profile and a large second segment.

nates in the simulations are shown in Table 3 . A color difference of 0.003 is considered very good by optical designers and is invisible for the human eye $[8,17]$. The measured color differences in the simulations are comfortably below this value, thus the color variation in the beam is eliminated.

\section{Conclusions}

We introduced an inverse method to design a TIR collimator that eliminates CoA variation for a point light source. This method improves the method introduced earlier in [6] by producing collimators that closely resemble standard collimators and at the same time 


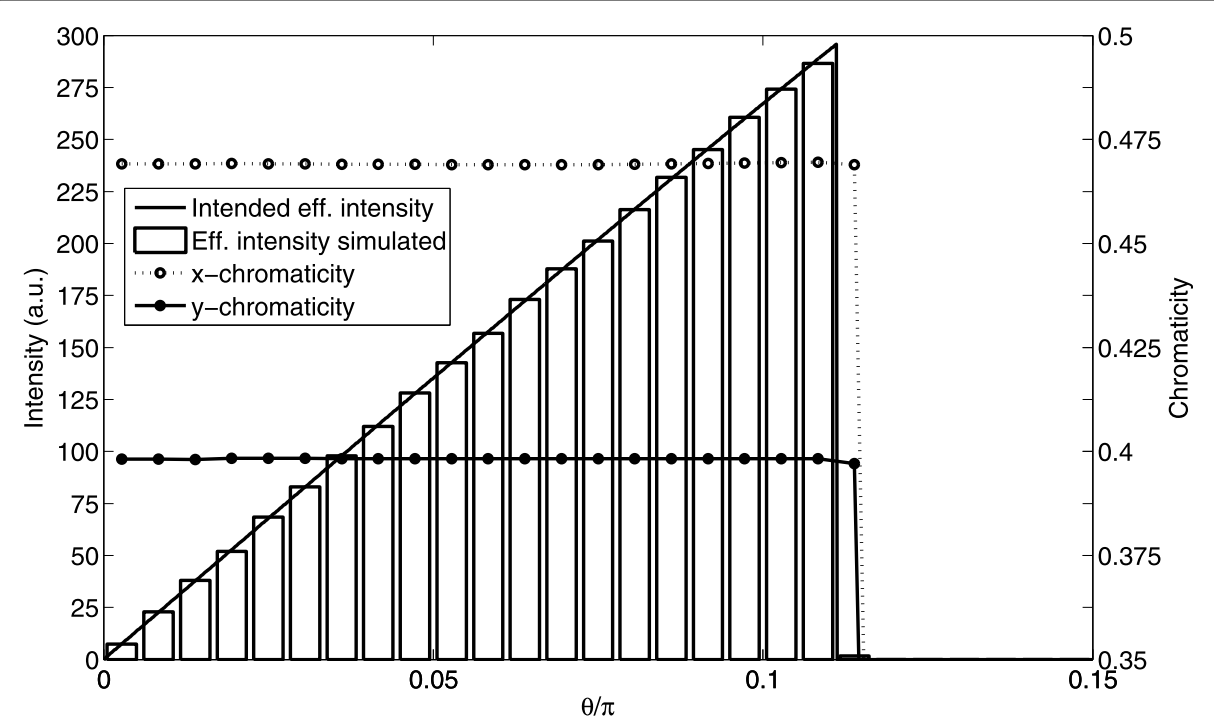

Figure 13 LightTools simulation results for the collimator with Block profile for LED02.

Table 3 Average chromaticity coordinates of the LEDs and the maximum difference with the chromaticity coordinates in the simulations

\begin{tabular}{llllll}
\hline Collimator & LED & Target $\boldsymbol{x}$ & $\max (|\boldsymbol{\Delta x}|)$ & Target $\boldsymbol{y}$ & $\max (|\boldsymbol{\Delta} \boldsymbol{y}|)$ \\
\hline Gaussian, small 2nd segment & LED16 & 0.4181 & $4 \cdot 10^{-4}$ & 0.3862 & $7 \cdot 10^{-4}$ \\
Gaussian, large 2nd segment & LED16 & 0.4181 & $4 \cdot 10^{-4}$ & 0.3862 & $1 \cdot 10^{-3}$ \\
Block profile & LED02 & 0.4691 & $4 \cdot 10^{-4}$ & 0.3985 & $5 \cdot 10^{-4}$ \\
\hline
\end{tabular}

have more parameters for optical design. In Section 3 we discussed which choices for these design parameters give meaningful results. In Section 4 we tested the method and verified the resulting collimators with Monte-Carlo raytracing using the software package LightTools. The simulations show color variations that are not visible with the human eye. Unfortunately, LEDs are too large to be treated as a point light source. In future research, we would like to extend this method to take the finite size of the light source into account using iterative methods such as described in for example $[18,19]$. This point source method will be an important building block in such an iterative method.

Competing interests

The authors declare that they have no competing interests.

Authors' contributions

The main idea in this paper was proposed by CP, who also prepared the manuscript initially. The proofs were elaborated by CP and JtTB. Technical assistance was provided by TT. All authors provided feedback on the manuscript, and read and approved the final manuscript.

\section{Author details}

${ }^{1}$ Centre for Analysis, Scientific computing and Applications (CASA), Technische Universiteit Eindhoven, P.O. Box 513, 5600 MB Eindhoven, The Netherlands. ${ }^{2}$ Philips Research, High Tech Campus 44, 5656 AE Eindhoven, The Netherlands. ${ }^{3}$ Philips Lighting, High Tech Campus 44, 5656 AE Eindhoven, The Netherlands.

Received: 13 December 2012 Accepted: 24 March 2014 Published: 03 Jun 2014

References

1. Haitz R, Jeffrey YT: Solid-state lighting: 'The case' 10 years after and future prospects. Phys Status Solidi A 2011, 208:17-29.

2. Schubert F: Light-Emitting Diodes. 2nd edition. Cambridge: Cambridge University Press; 2006. 
3. Wu H, Narendran N, Gu Y, Bierman A: Improving the performance of mixed-color white LED systems by using scattered photon extraction technique. In Light-Emitting Diodes; 2007. [Proc. SPIE, vol. 6669.]

4. Mueller GO: Luminescent ceramic for a light emitting device. United States patent US7361938 B2.

5. Wang K, Wu D, Chen F, Liu Z, Luo X, Liu S: Angular color uniformity enhancement of white light-emitting diodes integrated with freeform lenses. Opt Lett 2010, 35:1860-1862.

6. Prins C, ten Thije Boonkkamp C, Tukker T, IJzerman W: An inverse method for the design of TIR collimators to achieve a uniform color light beam. J Eng Math 2013, 81:177-190.

7. Maes M: Mathematical methods for reflector design. PhD thesis. University of Amsterdam; 1997.

8. Malacara D: Color Vision and Colorimetry. Theory and Applications. Bellingham: SPIE; 2002.

9. Wyszecki G, Stiles W: Color Science. Concepts and Methods, Quantitative Data and Formulae. 2nd edition. New York: Wiley; 2000

10. Bortz J, Shatz N: Generalized functional method of nonimaging optical design. In Nonimaging Optics and Efficient Illumination Systems III. Edited by Winston R, Benitez P; 2006. [Proc. SPIE, vol. 6338.]

11. Bortz J, Shatz N: Mathematical relationships between the generalized functional, edge-ray and sms design methods. In Nonimaging Optics and Efficient Illumination Systems III; 2010. [Nonimaging Optics: Efficient Design for Illumination and Solar Concentration, vol. 7785.]

12. Stöcker H: Taschenbuch mathematischer Formeln und moderner Verfahren. Thun: Verlag Harri Deutsch; 1993

13. ORA LightTools product website [http://www.opticalres.com/lt/ltprodds_f.html] [Accessed May 31, 2013].

14. Instrument Systems - Light measurement

[http://www.instrumentsystems.com/products/led-measurement-accessories/ledgon-goniophotometer/] [Accessed July 10, 2012].

15. Weisstein E: Gaussian function [http://mathworld.wolfram.com/GaussianFunction.html]

16. Weisstein E: Full width at half maximum [http://mathworld.wolfram.com/FullWidthatHalfMaximum.html]

17. MacAdam D: Visual sensitivities to color differences in daylight. J Opt Soc Am 1942, 32:247-274.

18. Zhang W, Liu Q, Gao H, Yu F: Free-form reflector optimization for general lighting. Opt Eng 2010, 49(6):063003.

19. Cassarly WJ: Iterative reflector design using a cumulative flux compensation approach. Opt Eng 2010, 7652:76522.

10.1186/2190-5983-4-5

Cite this article as: Prins et al.: An inverse method for color uniformity in white LED spotlights. Journal of Mathematics in Industry 2014, 4:5

\section{Submit your manuscript to a SpringerOpen ${ }^{\ominus}$ journal and benefit from:}

- Convenient online submission

- Rigorous peer review

- Immediate publication on acceptance

- Open access: articles freely available online

- High visibility within the field

- Retaining the copyright to your article 